\title{
Sequence similarity of HSP65 of Mycobacterium bovis BCG with SARS-CoV-2 spike and nuclear proteins: may it predict an antigen-dependent immune protection of BCG against COVID-19?
}

\author{
Paola Finotti ${ }^{1}$ (1)
}

Received: 23 September 2021 / Revised: 3 November 2021 / Accepted: 4 November 2021 / Published online: 9 November 2021

(c) Cell Stress Society International 2021

\begin{abstract}
The Bacillus Calmette-Guérin (BCG) vaccine is known to have protective effects not only against tuberculosis but also against other unrelated infectious diseases caused by different pathogens. Several epidemiological studies have also documented the beneficial influence of BCG vaccine in reducing both susceptibility to and severity of SARS-CoV-2 infection. The protective, non-specific effects of BCG vaccination would be related to an antigen-independent enhancement of the innate immunity, termed trained immunity. However, the knowledge that heat shock protein (HSP)65 is the main antigen of Mycobacterium bovis BCG prompted us to verify whether sequence similarity existed between HSP65 and SARS-CoV-2 spike (S) and nuclear $(\mathrm{N})$ proteins that could support an antigen-driven immune protection of BCG vaccine. The results of the in silico investigation showed an extensive sequence similarity of HSP65 with both the viral proteins, especially SARS-CoV-2 S, that also involved the regions comprising immunodominant epitopes. The finding that the predicted B cell and CD4 ${ }^{+} \mathrm{T}$ cell epitopes of HSP65 shared strong similarity with the predicted B and T cell epitopes of both SARS-CoV-2 S and N would support the possibility of a cross-immune reaction of HSP65 of BCG with SARS-CoV-2.
\end{abstract}

Keywords Bacillus Calmette-Guérin BCG vaccine $\cdot$ SARS-CoV-2 $\cdot$ Heat shock proteins · Sequence analysis · Epitope mapping

\section{Introduction}

Since the beginning of the ongoing COVID-19 pandemic, much effort has been spent to identify the regions of the viral proteins targeted by neutralizing antibodies. Studies dealing with the functions and structures of neutralizing antibodies isolated from COVID-19 patients have shown that these antibodies are prevalently, but not exclusively, directed against the spike (S) protein, responsible for the virus entry into host cells (Kared et al. 2021; Shrock et al. 2020).

A detailed analysis of the humoral immunity of COVID19 patients allowed to identify and characterize the immune dominant regions in both $\mathrm{S}$ and nuclear $(\mathrm{N})$ proteins of SARS-CoV-2 (Shrock et al. 2020). Epitopes of S protein

Paola Finotti

paola.finotti@unipd.it

1 Department of Pharmaceutical and Pharmacological Sciences, University of Padua, Building "C," Largo E. Meneghetti 2, 35135 Padua, Italy were found not only in the region of the receptor binding domain (RBD) but also in regions comprised in the sequence 617-1200 (Shrock et al. 2020; Morgenlander et al. 2021) in which a cluster of epitopes of immune relevance was specifically identified around the sequence 766-840 (Shrock et al. 2020). Antibodies targeting the epitopes in the RBD and inhibiting the binding of S protein to ACE2 receptor have potent neutralizing activity, being thus the focus in the development of therapeutic interventions for COVID-19 (Henderson 2020), including the currently used vaccines that incorporate $\mathrm{S}$ protein to elicit humoral immunity (Castells and Phillips 2020).

Although it is well recognized that COVID-19 vaccines can induce a SARS-CoV-2-specific T cell response similar with that yielded by the natural infection, several experimental data now indicate that many $\mathrm{T}$ cell targets of SARS$\mathrm{CoV}-2$ exist besides those identified in S protein (Grifoni et al. 2020a; Chi et al. 2020). The observation that antibodies isolated from recovered SARS-CoV-2 patients displayed neutralizing activity without inhibiting the virus binding at its receptor supported the view that other mechanisms of 
neutralization of SARS-CoV-2 could exist besides those involving the immunodominant targets of $\mathrm{S}$ protein (Chi et al. 2020). For this reason, it has been proposed that other structural protein antigens, mostly $\mathrm{N}$ protein, should be included in candidate vaccines to better mimic the humoral response elicited in COVID-19 patients (Grifoni et al. 2020a; Chi et al. 2020). This highlights the need for any therapeutic or preventive intervention against COVID-19 to induce a stable and broad immune response aimed at preventing resistance mutations of the virus often linked to the use of a single therapeutic agent (Chi et al. 2020).

The population studies conducted so far to investigate the factors that might affect the susceptibility to and the clinical course of SARS-CoV-2 infection showed that the Bacillus Calmette-Guérin (BCG) vaccination, besides having a well-known protective effect against tuberculosis, could also confer significant protection against $\mathrm{CoV}-2$ infection by reducing the spreading and severity of the disease (Curtis et al. 2020; Gupta 2020; Escobar et al. 2020; Berg et al. 2020; Ytbarek et al. 2020; Rivas et al. 2021). It is widely accepted that the protective effects that BCG vaccine has against many, apparently unrelated infectious agents, especially those affecting the respiratory tract, are due to an antigen-independent $\mathrm{B}$ and $\mathrm{T}$ cell activation and to long-term changes in innate immune memory, termed trained immunity (Curtis et al. 2020; Escobar et al. 2020; O'Neill and Netea 2020; Sidi 2020). Moreover, efficacy of BCG vaccination on trained immunity is reported to last even for years (Escobar et al. 2020; Rivas et al. 2021). The possibility that BCG vaccine could also induce an adaptive immune response has not been investigated thoroughly so far, and only limited information exists suggesting a relationship between BCG vaccination and generation of $\mathrm{T}$ cells cross-reacting with SARS-CoV-2 (Tomita et al. 2020).

However, to prove that the protective immunity induced by BCG against SARS-CoV-2 recognizes an antigendependent mechanism, it is necessary to establish which protein, among those identified in the Mycobacterium bovis, possesses a higher immunogenic potential. Previous studies performed to isolate and characterize protein antigens from Mycobacterium bovis BCG had identified the 65-kDa HSP as the main antigen, common to a variety of mycobacterial species, including the Mycobacterium tuberculosis (Thole et al. 1985). HSP65 is considered the major immunogenic component of Mycobacterium bovis BCG, responsible for inducing a potent, antigen-specific $\mathrm{CD} 4^{+}$and $\mathrm{CD} 8^{+} \mathrm{T}$ cell activation (Ottenhoff et al. 1988). It is known that besides the role displayed in the intra-cellular, physiological setting, HSPs become potent inducers of both innate and antigenspecific immunity when liberated in the extra-cellular environment (Wallin et al. 2002; Segal et al. 2006). Moreover, molecular mimicry has been postulated to occur between some SARS-CoV-2 proteins and human HSPs that might account for specific adverse effects of SARS-CoV-2 in the vascular bed (Marino Gammazza et al. 2020).

These considerations prompted us to hypothesize that cross-immunity of BCG vaccine with SARS-CoV-2 might occur due to crucial similarity between the sequence of HSP65 and those of viral proteins, in particular S and N proteins. More specifically, if sequence similarity involved sequences of the viral proteins identified as those with high immunogenic potential, then, stimulation of the immune system by BCG vaccine might induce the production of antibodies also able to recognize and bind these immunogenic sequences, thus contributing to the immune protection against the virus. As a preliminary approach to address this question, we performed a thorough in silico analysis of sequence similarity between HSP65 of Mycobacterium bovis and both $\mathrm{S}$ and $\mathrm{N}$ proteins of SARS-CoV-2. We also tested whether, based on sequence similarity with the $\mathrm{B}$ and $\mathrm{T}$ cell epitopes identified in $\mathrm{S}$ and $\mathrm{N}$ proteins, the predicted immunodominant B and T cell epitopes of HSP65 could support an antigen-dependent mechanism in the immune response of BCG vaccine.

\section{Materials and methods}

\section{Protein sequence analysis}

Protein sequences of HSP65 (65 kDa antigen, CH602 MYCBO, accession number P0A521), S protein CoV-2 (SPIKE_SARS2; P0DTC2), and N protein CoV-2 (NCAP_ SARS2; P0DTC9) were retrieved from UniProtKB/SwissProt (http://www.uniprot.org). Sequence similarities were analyzed in pair-wise alignment using two progressive alignment programs: T-Coffee (three-based consistency objective function for alignment evaluation package) package that combines different sequence-alignment programs (Notredame et al. 2000) and the Clustal Omega program (UniProtKB). The two programs use different algorithms in the sequence alignment so that different similar sequences can be detected in the same protein. Thus, both methods were used to detect as many similar sequences as possible. Only the sequences of at least eight consecutive amino acid residues without any gap and with the highest consistency score were considered and recorded.

\section{Prediction of the B and T cell epitopes}

Immune Epitope Database (IEDB Analysis Resource, http// tools.iedb.org/main/) was used to predict immune epitopes in HSP65 and CoV-2 S and N proteins. B cell epitopes were predicted by means of Bepipred Linear Epitope Prediction 2.0 tool (http://tools.iedb.org/bcell/) whereas peptide binding to MHC class II molecules $\left(\mathrm{CD}^{+}{ }^{+} \mathrm{T}\right.$ cell predicted 
epitopes) was investigated using a tool that combines different methods (IEDB recommended 2.22 in http://tools.iedb. org/mhcii/) (Wang et al. 2008). The 7-allele method was chosen for identifying top immunodominant peptides. The method makes prediction based on the median consensus percentile ranks for the seven most represented DR alleles.

\section{Results}

\section{High degree of sequence similarity between HSP65 and SARS-CoV-2 spike protein}

Results of the pair-wise alignment of HSP65 with CoV-2 $\mathrm{S}$ revealed an impressive degree of similarity between the two proteins that shared 468 similar amino acid residues (87\% of the HSP65 sequence) and 149 identical residues

(Fig. 1A). Interestingly, the sequences of CoV-2 S identified as similar with T-Coffee did not overlap those found with Clustal Omega. Since both methods detected an extensive sequence similarity in HSP65, the different procedures of alignment allowed us to discover that almost the entire HSP65 sequence was covered by a great number of similar, distinct sequences of CoV-2 S (Fig. 1A). In particular, the first 320 residues of HSP65 showed an almost uninterrupted similarity with sequences of $\mathrm{CoV}-2 \mathrm{~S}$ that also encompassed immunodominant epitopes, such as the regions 404-588 (receptor binding domain), 617-672, 766-835, and 813-868 (Shrock et al. 2020; Morgenlander et al. 2021) (Fig. 1B). The two separate methods of alignment also revealed the high degree of similarity displayed by single sequences of CoV-2 $\mathrm{S}$ with separate sequences of HSP65, as well as the crosssimilarity of separate sequences of CoV-2 S with single sequences of HSP65. Thus, the 405-588 long sequence of $\mathrm{CoV}-2 \mathrm{~S}$, containing epitopes of the RBD, showed similarity to both the sequence 101-176 and three separate sequences

A

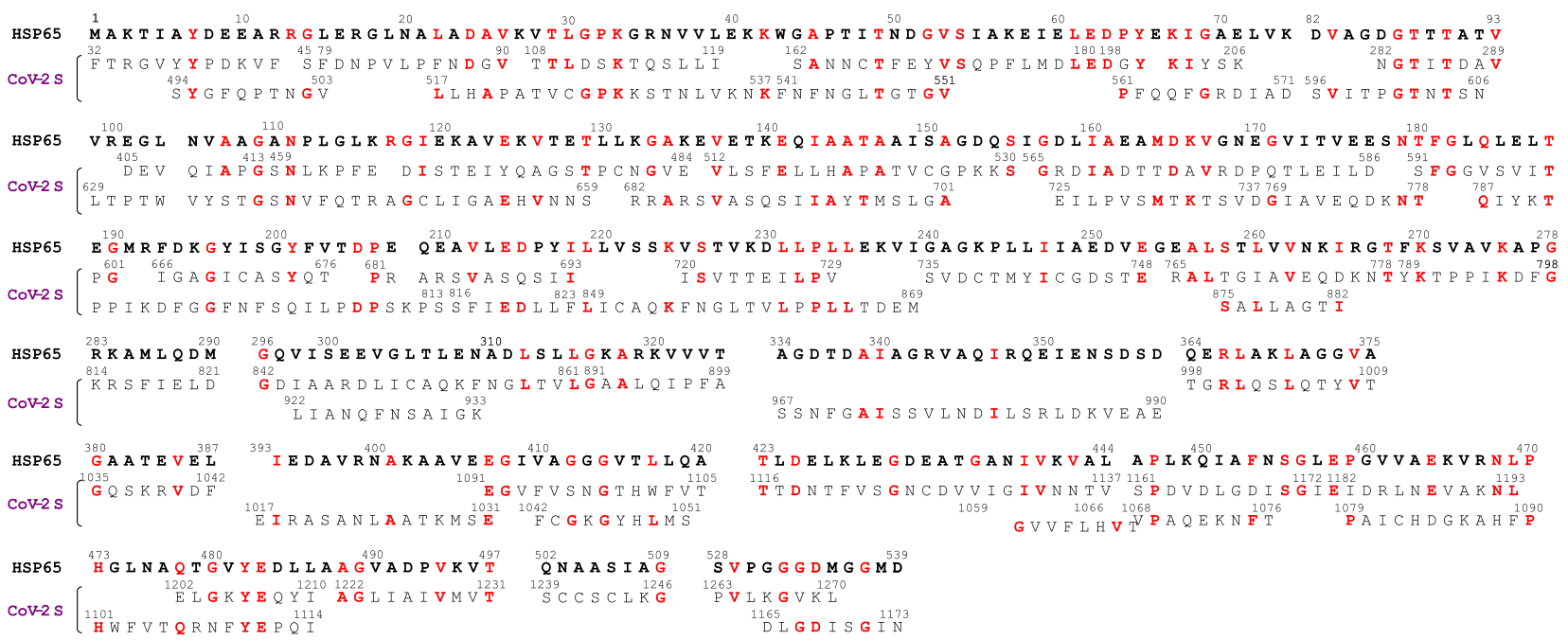

B

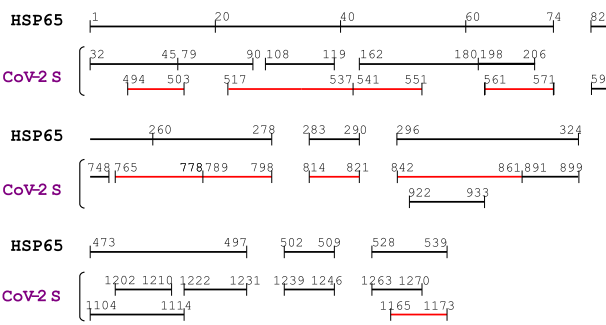

Fig. 1 Sequence similarity between HSP65 of Mycobacterium bovis BCG and the spike (S) protein of SARS-CoV-2. Pair-wise alignment of the protein sequences was performed as specified in "Materials and methods." A All the sequences of S protein (CoV-2 S) with similarity to HSP65, detected with both T-Coffee (upper line) and Clustal Omega (lower line), are reported below the corresponding sequences of HSP65 showing the amino acid residues (one-letter code) and sequence numbering (starting from the amino terminus of each protein). In red are the identical residues in both HSP65 and CoV-2 S.
B The same sequences, as in $\mathbf{A}$, are presented in graphical form to visualize the portions of $\mathrm{CoV}-2 \mathrm{~S}$ with similarity to HSP65. In red are the sequences identified experimentally as epitopes of CoV-2 S (see "Results"). One-letter code abbreviations for amino acid residues are the following: A, alanine; C, cysteine; D, aspartic acid; E, glutamic acid; F, phenylalanine; G, glycine; $\mathrm{H}$, histidine; I, isoleucine; $\mathrm{K}$, lysine; L, leucine; M, methionine; N, asparagine; P, proline; $\mathrm{Q}$, glutamine; $\mathrm{R}$, arginine; $\mathrm{S}$, serine; $\mathrm{T}$, threonine; $\mathrm{V}$, valine; $\mathrm{W}$, tryptophan; Y, tyrosine 
located in the first 74 residues of HSP65 (Fig. 1B). The sequences encompassing the CoV-2 S 766-840, identified as a region of high density of distinct epitopes (Shrock et al. 2020) and containing two epitope clusters near the residues 770 and 815 , displayed similarity with the sequences 171-218 and 255-290 of HSP65 (Fig. 1A). The CoV-2 S sequence 813-868 identified as a target of COVID-19 convalescent plasma (Morgenlander et al. 2021) was similar to two distinct regions of HSP65 comprising residues 219-239 and 296-315 (Fig. 1A). Likewise, the immunodominant region $405-586$ of CoV-2 $\mathrm{S}$ with similarity to sequences 1-74 and 101-177 of HSP65 also displayed similarity to distinct sequences located in the first 206 residues of CoV-2 $\mathrm{S}$, as well as with the CoV-2 S sequences 629-659, 682-701, 725-737, and 769-778 (Fig. 1B). CoV-2 S 629-659 is within the 617-672 region identified as the epitope targeted by antibodies of COVID-19 convalescent plasma (Morgenlander et al. 2021). The result that individual sequences of HSP65 showed strong cross-similarity with separate regions of CoV-2 S, regardless of whether these comprise immunodominant epitopes, would imply that immune responses against these HSP65 regions might also target CoV-2 S sequences outside those known as main targets of neutralizing antibodies.

Although fewer Cov-2 S sequences showed similarity to the last 200 residues of HSP65, these sequences nevertheless regarded the CoV-2 S region between residues 967 and 1270 (Fig. 1A) comprising the epitopes 1121-1176 and 1149-1204 targeted by plasma of COVID-19 patients (Morgenlander et al. 2021) (Fig. 1B).

\section{Crucial sequence similarity between HSP65 and SARS-CoV-2 nuclear protein}

In the alignment of HSP65 with CoV-2 N, a smaller number of sequences and amino acid residues (186, of which 46 identical) were detected in HSP65 with similarity to CoV-2 N. It was also observed that similarity was unevenly distributed along the sequence of both proteins, with many long gaps between one and the other similar sequence (Fig. 2A). However, at variance with what noted with CoV-2 S, the most part of the sequences of CoV-2 $\mathrm{N}$ with similarity to HSP65 were identified by both T-Coffee and Clustal Omega (Fig. 2A, underlined sequences), a finding that underscores the relevance of this similarity. Moreover, nine out of the eleven CoV-2 $\mathrm{N}$ sequences with similarity to HSP65 were also recognized as those encompassing the epitopes 25-56, 85-140, 151-175, 169-224, 225-280, 337-392, and 363-420 (Shrock et al. 2020; Morgenlander et al. 2021) (Fig. 2B).

\section{Identification of HSP65 sequences as potential B and $T$ cell epitopes}

To validate the hypothesis that the significant sequence similarity between the two CoV-2 proteins and HSP65 could support cross-immunity, the investigation was performed to verify which and how many of the similar sequences found in HSP65 might contain B and T cell epitopes overlapping those predicted to occur in both CoV-2 S and N (Grifoni et al. 2020b). Twenty-eight peptides were identified in HSP65 as B cell epitopes, and seventeen of these showed the highest score of similarity with sequences of both CoV-2 $\mathrm{S}$ and $\mathrm{N}$ already reported to be $\mathrm{B}$ cell epitopes (Grifoni et al. 2020b) (Fig. 3A, boxed sequences). For some of these HSP65 epitopes, the similarity was confirmed with
A

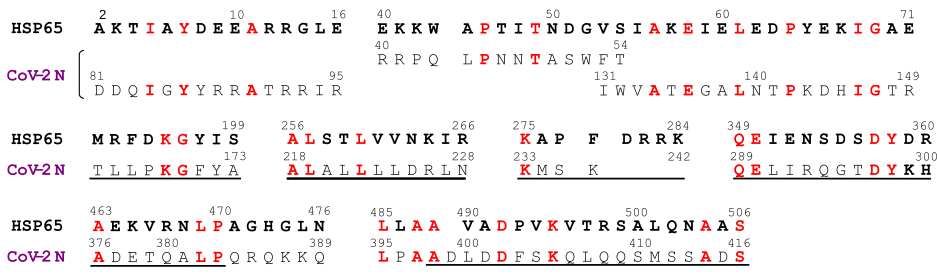

B
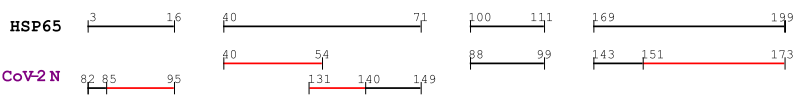

Fig. 2 Sequence similarity between HSP65 of Mycobacterium bovis $\mathrm{BCG}$ and nucleoprotein $(\mathrm{N})$ of SARS-CoV-2. The pair-wise alignment of HSP65 with nucleoprotein $(\mathrm{Cov}-2 \mathrm{~N})$ was performed as specified in "Materials and methods." A All the sequences of Cov-2 N with similarity to HSP65, detected with both T-Coffee (upper line) and Clustal Omega (lower line), are reported below the correspond-

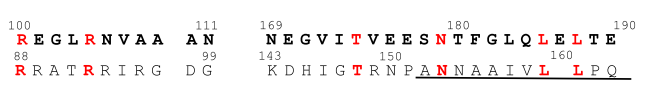

${ }^{413}$ G V T L L Q A A P T L D E L K LE G D E A T G A

$\frac{335 \text { A IKLDDKDPNFKDQVILLNKHIDA }}{350}$
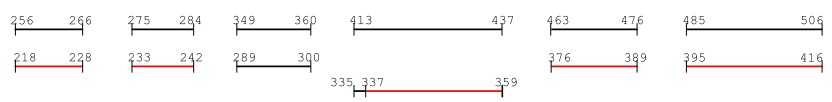

ing sequences of HSP65, as in Fig. 1. Underlined are the sequences of CoV-2 $\mathrm{N}$ identified with both methods as similar with HSP65. In red are the amino acid residues common to both proteins. B The same sequences, as in $\mathbf{A}$, are presented in graphical form to visualize the portions of $\mathrm{CoV}-2 \mathrm{~N}$ with similarity to HSP65. In red are the sequences identified experimentally as epitopes of CoV-2 N 
A

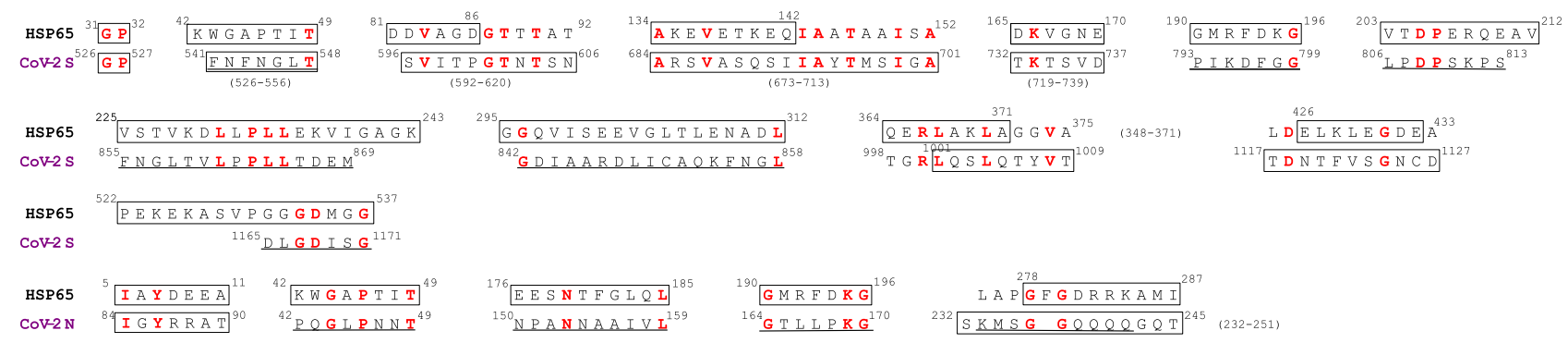

B

\begin{tabular}{|c|c|c|c|}
\hline \multirow{2}{*}{ HLA-DR Alleles } & HSP65 sequence & Percentile rank & $I C_{50}(\mathrm{nM})$ \\
\hline & $215-229$ & 1.10 & "33 \\
\hline \multirow{3}{*}{$B 1 * 15: 01$} & $214-228$ & 1.30 & "33 \\
\hline & $216-230$ & 1.30 & "49 \\
\hline & $213-227$ & 2.50 & "35 \\
\hline \multirow{8}{*}{$B 3 * 02: 02$} & $215-229$ & 2.80 & " "- \\
\hline & $214-228$ & 3.60 & " "- \\
\hline & $216-230$ & 3.20 & ""- \\
\hline & $217-231$ & 2.33 & " "- \\
\hline & $257-271$ & 2.40 & ""- \\
\hline & $256-270$ & $\begin{array}{l}2.40 \\
2.60\end{array}$ & " "- \\
\hline & $258-272$ & 3.20 & " - \\
\hline & $255-269$ & 3.50 & 141 \\
\hline \multirow{7}{*}{$B 1 * 03: 01$} & " "1-15 & 3.20 & 113 \\
\hline & $"=2-16$ & 3.30 & 113 \\
\hline & $514-528$ & 3.20 & "93 \\
\hline & $513-527$ & 3.50 & "93 \\
\hline & $515-529$ & 3.90 & "93 \\
\hline & $511-525$ & 3.90 & "95 \\
\hline & $512-526$ & 3.90 & "93 \\
\hline \multirow{5}{*}{$B 4 * 01: 01$} & $443-457$ & 2.90 & 145 \\
\hline & $444-458$ & 2.90 & 144 \\
\hline & $446-460$ & 3.0 & 149 \\
\hline & $445-459$ & 3.0 & 147 \\
\hline & $442-456$ & 3.1 & 152 \\
\hline$B 3 * 01: 01$ & $188-202$ & 3.50 & 141 \\
\hline
\end{tabular}

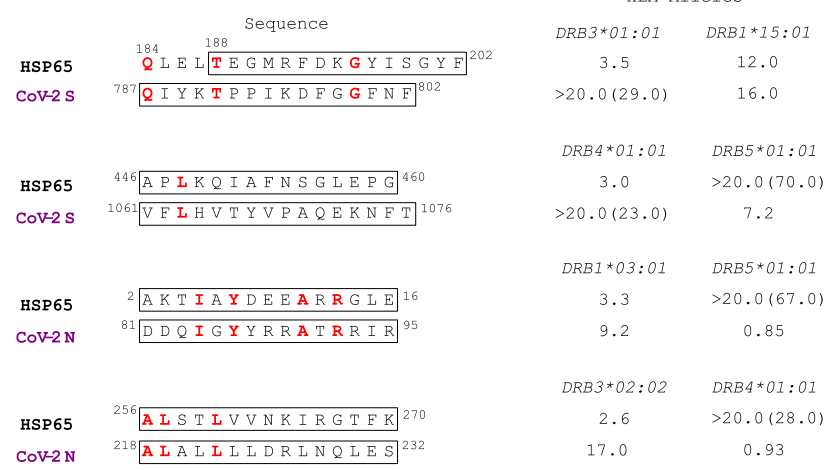

Fig. 3 Predicted B and T cell epitopes of HSP65 with similarity to epitopes of both CoV-2 S and CoV-2 N. A B cell epitopes of HSP65, CoV-2 S, and CoV-2 N were found using Bepipred Linear Epitope Prediction 2.0 tool in IEDB Analysis Resource (http:// tools.iedb.org/main). Only the sequences of HSP65, CoV-2 S, and CoV-2 $\mathrm{N}$ are shown that entirely or partially (in this case, the entire sequence is indicated below, in parenthesis) contain the predicted B cell epitopes (boxed sequences). These sequences are among those identified as similar in the protein alignments (Figs. 1 and 2). Underlined sequences correspond to the epitopes of CoV-2 S and CoV-2 N

the sequences of CoV-2 $\mathrm{S}$ and $\mathrm{N}$ identified experimentally as targets of neutralizing antibodies (Fig. 3A, underlined sequences). Single residues identified as B cell epitopes in HSP65 were E124 and E128, T188, and I198. E124 and T188 of HSP65 are identical, respectively, to E654 and T791 in CoV-2 S (Fig. 1A) and both these residues are comprised in sequences referred as epitopes. The sequence 482-485 (YEDL) in HSP65, with similarity to two separate sequences in CoV-2 S (1206-1209 and 1110-1113) (Fig. 1A), is also a predicted B cell epitope.

Although numerous peptides of HSP65 were identified as potential $\mathrm{CD}^{+} \mathrm{T}$ cell epitopes for having a median consensus percentile rank $\leq 20$ (data not shown), only the topranking peptides with the lowest percentile rank $(\leq 4.0)$, i.e., highest binding affinity to HLA-DR alleles $\left(\mathrm{IC}_{50} \leq 500 \mathrm{nM}\right)$, were chosen for further analysis (Fig. 3B, left side).

determined experimentally (Figs. 1 and 2, red lines in $\mathbf{B}$ panels). B Predicted immunodominant epitopes of HSP65 with the best binding affinity to a restricted panel of HLA class II molecules (the seven more represented in the general population). The HSP65 top peptides were screened for similarity with the panel of the predicted $\mathrm{CD}^{+}$ epitopes in both CoV-2 S and CoV-2 N and those found similar are shown as boxed sequences together with the HLA-DR alleles for which each predicted epitope displays the best binding affinity (values of median percentile rank indicated below the HLA alleles)

Overlapping peptides in the HSP65 sequence 213-231 displayed high binding affinity to two HLA-DR alleles, whereas binding to one single allele was noted for the other epitopes. Among the best immunodominant epitopes, four turned out to display significant similarity to two sequences of both CoV-2 S and CoV-2 N (Fig. 1A) predicted to be $\mathrm{CD}^{+}{ }^{+}$epitopes with high affinity to five HLA-DR alleles (Fig. 3B, right side). A higher binding affinity was displayed by the predicted epitopes of HSP65, compared with similar epitopes of both CoV-2 S and CoV-2 N, to the HLA alleles $D R B 3 * 01: 01, D R B 1 * 15: 01, D R B 4 * 01: 01, D R B 1 * 03: 01$, and $D R B 3 * 02: 02$. Three epitope sequences of HSP65 corresponded to those already identified in the pair-wise alignments (Figs. 1 and 2) as similar with the sequences of CoV-2 S (787-802) and CoV-2 N (81-95 and 218-232) indicated as target of neutralizing antibodies. 


\section{Discussion}

The results of several epidemiological studies have demonstrated a consistent association between BCG vaccination and protection from severe COVID-19, a reason for which efficacy of BCG vaccination against SARS-CoV-2 is currently being tested in many controlled clinical trials (O'Neill and Netea 2020). Ample epidemiological evidence also exists to show that BCG vaccination is safe and has broad protective effects against a range of various respiratory infections, not only in children but also in the elderly (Giamarellos-Bourboulis et al. 2020). The mechanism underlying the numerous and non-specific protective effects of BCG vaccine has been the subject of an intense debate. It has been observed that the molecular similarity between BCG and viral antigens could not explain so many diverse effects, and the largely accepted interpretation nowadays is that BCG vaccine can enhance an antigenindependent innate immunity (Curtis et al. 2020; Gupta 2020; O'Neill and Netea 2020). However, the possibility that an antigen-driven activation of the adaptive immune response is responsible for the complex and long-lived protection of BCG vaccine should also be considered on the grounds that HSP65 is recognized as the main antigen among all the proteins of Mycobacterium bovis.

In this work, we tested whether sequence similarity between HSP65 and both CoV-2 S and CoV-2 N could support an antigen-dependent mechanism in the immune protection elicited by BCG vaccine against SARS-CoV-2. The in silico analysis revealed a striking similarity between HSP65 and CoV-2 S such that almost the entire sequence of HSP65 was recognized by multiple and distinct regions of CoV-2 $\mathrm{S}$ including those comprising all the immunodominant epitopes identified so far (Shrock et al. 2020; Morgenlander et al. 2021) (Fig. 1). The application of two methods that use different alignment procedures to find sequence similarity allowed us to discover that many CoV-2 S sequences with similarity to separate regions of HSP65 also showed cross-similarity with other distant sequences of CoV-2 S (Fig. 1B). The cross-similarity shared by both epitope and non-epitope regions of $\mathrm{CoV}-2$ $\mathrm{S}$ with the same HSP65 sequences would support the view that the humoral response against HSP65 might also be extended to a broad range of different, non-epitope regions of CoV-2 S. In addition, the significant sequence similarity displayed by HSP65 with CoV-2 N, that regarded sequences for the most part identified as epitopes (Fig. 2), and, importantly, was detected by both the alignment methods, would suggest that also CoV-2 $\mathrm{N}$ could be a target of a cross-immune reaction induced by HSP65.

The evidence accumulated so far has demonstrated that many potential $\mathrm{T}$ cell targets exist in SARS-CoV-2 other than those of CoV-2 S (Kared et al. 2021; Grifoni et al. 2020a; Chi et al 2020), corroborating the idea that future COVID-19 vaccines should include not only $S$ protein but other structural and non-structural antigen proteins to better mimic the natural $\mathrm{T}$ cell response observed in disease (Kared et al. 2021; Grifoni et al. 2020a). To satisfy the requisite of an effective COVID-19 vaccine, BCG should thus contain epitopes that are targets of $\mathrm{B}$ and $\mathrm{T}$ cells. The finding that HSP65 shared with CoV-2 S and $\mathrm{N}$ a number of similar sequences predicted to be $\mathrm{B}$ cell epitopes (Fig. 3A), together with the result that some of the HSP65 sequences identified as class II immunodominant epitopes also shared sequence similarity and high binding affinity with predicted $\mathrm{CD} 4^{+}$epitopes in CoV-2 $\mathrm{S}$ and N (Fig. 3B), would further support the possibility that HSP65 of BCG can induce a specific cross-immune response to SARS-Cov-2.

Whether BCG can thus be used as an additional effective vaccine against SARS-CoV-2 cannot be inferred from the present investigation and only additional experimental studies, together with data of ongoing clinical trials, can confirm the validity of this proposal. Our results can nevertheless offer a plausible explanation for the observed beneficial effects of BCG vaccine against SARS-CoV-2 and help understand how HSP65 of BCG might also induce an adaptive immunity against different infectious agents. HSPs display their physiological role as chaperones in the intracellular environment, whereas they become highly immunogenic when expressed extra-cellularly (Wallin et al. 2002; Segal et al. 2006). One must also consider that a high degree of homology exists in the phylogenesis among the members of the same HSP class, a reason for which HSP65 of Mycobacterium bovis, besides being the antigen common to several different mycobacterial species (Thole et al. 1985), also shows strong similarity with human HSP60. Molecular mimicry also exists between many HSPs and the proteins of different bacterial agents (Mayr et al. 1999; Cappello et al. 2009) and human viruses (Bason et al. 2003). Thus, it is tempting to speculate that the immune response elicited by the immunogenic sequences of HSP65 of BCG with similarity to sequences of bacterial/viral proteins, other than those of mycobacteria, might also effectively target the pathogens responsible for the infections against which BCG vaccination has proven to be protective.

Acknowledgements I would like to thank Dr. Pietro Veronesi per his helpful assistance in drafting the figures.

\section{References}

Bason C, Corrocher R, Lunardi C, Puccetti P, Olivieri O, Girelli D, Navone R, Beri R, Millo E, Margonato A, Martinelli N, Puccetti A (2003) Interaction of antibodies against cytomegalovirus with heat 
shock protein 60 in pathogenesis of atherosclerosis. The Lancet 362:1971-1977

Berg MK, Yu Q, Salvador CE, Melani I, Kitayama S (2020) Mandated bacillus Calmette-Guérin (BCG) vaccination predicts flattened curves for the spread of COVID-19. SciAdv 6:eabc1463

Cappello F, Conway de Macario E, Di Felice V, Zummo G, Macario AJL (2009) Chlamydia trachomatis infection and anti-Hsp60 immunity: the two sides of the coin. PLoS Pathogens 5 (8): e1000552

Castells MC, Phillips EJ (2020) Maintaining safety with SARS-CoV-2 vaccines. N Engl J Med. https://doi.org/10.1056/NEJMra2035343

Chi X, Yan R, Zhang J, Zhang G, Zhang Y, Hao M, Zhang Z, Fan P, Dong Y, Yang Y, Chen Z, Guo Y, Zhang J, Li Y, Song X, Chen Y, Xia L, Fu L, Hou L, Xu J, You C, Li J, Zhao Q, Chen W (2020) A neutralizing human antibody binds to the $\mathrm{N}$-terminal domain of the spike protein of SARS-CoV-2. Science 369:650-655

Curtis N, Sparrow A, Ghebreyesus TA, Netea MG (2020) Considering BCG vaccination to reduce the impact of COVID-19. The Lancet 395:1545

Escobar LE, Molina-Cruz A, Barillas-Mury C (2020) BCG vaccine protection from severe coronavirus disease 2019 (COVID-19). Proc Natl Acad Sci USA 117(30):17720-17726

Giamarellos-Bourboulis EJ, Tsilika M, Moorlag S, Antonakos N, Kotsaki A, Dominguez-Andrés J, Kyriazopoulou E, Gkuogianni T, Adami ME, Damoraki G, Koufargyris P, Karageorgos A, Bolanou A, Koenen H, van Crevel R, Droggiti DI, Renieris G, Papadopoulos A, Netea MG (2020) Activate: randomized clinical trials of BCG vaccination against infection in the elderly. Cell $183: 315-323$

Grifoni A, Weiskopf D, Ramirez SI, Mateus J, Dan JM, Rydyznski Moderbacher C, Rawlings SA, Sutherland A, Premkumar L, Jadi RS, Marrama D, de Silva A, Frazier A, Carlin AF, Greenbaum JA, Peters B, Krammer F, Smith DM, Crotty S, Sette A (2020a) Targets of T cell responses to SARS-CoV-2 coronavirus in humans with COVID-19 disease and unexposed individuals. Cell 181:1489-1501

Grifoni A, Sidney J, Zhang Y, Scheuermann RH, Peters B, Sette A (2020b) A sequence homology and bioinformatic approach can predict candidate targets for immune responses to SARS-CoV-2. Cell Host Microbe 27:671-680

Gupta PK (2020) New disease old vaccine: is recombinant BCG vaccine an answer for COVID-19? Cell Immunol 356:104187

Henderson JP (2020) What are protective antibody responses to pandemic SARS-CoV-2? J Clin Invest 130(12):6232-6234

Kared H, Red AD, Bloch EM, Bonny TS, Sumatoh H, Kairi F, Carbajo D, Abel B, Newell EW, Bettinotti MP, Benner SE, Patel EU, Littlefield K, Laeyendecker O, Shoham S, Sullivan D, Casadevall A, Pekosz A, Nardin A, Fehlings M, Tobian AAR, Quinn TC (2021) SARS-CoV-2-specific CD8 ${ }^{+} \mathrm{T}$ cell responses in convalescent COVID-19 individuals. J Clin Invest 131(5):e145476. https:// doi.org/10.1172/JCI45476

Marino Gammazza A, Légaré S, Lo Bosco G, Fucarino A, Angileri F (2020) Conway de Macario E, Macario AJL, Cappello F. Cell Stress Chaperones 25:737-741

Mayr M, Metzler B, Kiechl S, Willeit J, Schett G, Xu Q, Wick G (1999) Endothelial cytotoxicity mediated by serum antibodies to heat shock proteins of Escherichia coli and Chlamidia pneumoniae. Circulation 99:1560-1566

Morgenlander W, Henson S, Monaco DR, Chen A, Littlefield K, Bloch EM, Fujimura E, Ruczinski I, Crowley AR, Natarajan H, Butler SE, Weiner JA, Li MZ, Bonny TS, Benner SE, Balagopal A,
Sullivan D, Shoham S, Quinn TC, Eshleman SH, Casadevall A, Redd AD, Laeyendecker O, Ackerman ME, Pekosz A, Elledge SJ, Robinson M, Tobian AA, Larman HB (2021) Antibody responses to endemic coronaviruses modulate COVID-19 convalescent plasma functionality. J Clin Invest 131(7)e146927. https://doi. org/10.1172/JCI146927

Notredame C, Higgins DG, Heringa J (2000) T-Coffee: a novel method for fast and accurate multiple sequence alignment. J Biol Mol 302(1):205-217

O'Neill LAJ, Netea MG (2020) BCG-induced trained immunity: can it offer protection against COVID-19? Nat Rev Immunol 20:335-337

Ottenhoff THM, Kale B, van Embden JDA, Thole JER, Kiessling R (1988) The recombinant 65-kDa heat shock protein of Mycobacterium bovis bacillus Calmette-Guérin/M. Tubercolosis is a target molecule for $\mathrm{CD}^{+}{ }^{+}$cytotoxic $\mathrm{T}$ lymphocytes that lyse human monocytes. J Exp Med 168:1947-1952

Rivas MN, Ebinger JE, Wu M, Sun N, Braun J, Sobhani K, Van Eyk JE, Cheng S, Arditi M (2021) BCG vaccination history associates with decreased SARS-CoV-2 seroprevalence across a diverse cohort of health care workers. J Clin Invest 131(2):e145157

Segal BH, Wang X-Y, Dennis CG, Youn R, Repasky EA, Manjili MH, Subjeck JR (2006) Heat shock proteins as vaccine adjuvants in infections and cancer. Drug Disc Today 11(11/12):534-540

Shrock E, Fujimura E, Kula T, Timms RT, Lee I-H, Leng Y, Robinson ML, Sie BM, Li MZ, ChenY, Logue J, Zuiani A, McCulloch D, Lelis FJN, Henson S, Monaco DR, Travers M, Habibi S, Clarke WA, Caturegli P, Laeyendecker O, Piechocka-Trocha A, Li JZ, Khrati A, Chu HY, MGH COVID-19 Collection \& Processing Team, Villani A-C, Kays K, Goldberg MB, Hacohen N, Filbin MR, Yu XG, Walker BD, Wesemann DR, Larman HB, Lederer JA, Elledge SJ (2020) Viral epitope profiling of COVID-19 patients reveals cross-reactivity and correlates of severity. Science 370(6520): eabd4250. https://doi.org/10.1126/science.abd4250

Sidi GR (2020) Could BCG be used to protect against COVID-19? Nat Rev Urol 17:316-317

Thole JER, Dauwerse HG, Das PK, Groothuis DG, Schouls LM, van Embden JDA (1985) Cloning of Mycobacterium bovis BCG DNA and expression of antigens in Escherichia coli. Infect Immun 50(3):800-806

Tomita Y, Sato R, Ikeda T, Sakagami T (2020) BCG vaccine may generate cross-reactive $\mathrm{T}$ cells against SARS-CoV-2: in silico analyses and a hypothesis. Vaccine 38:6352-6356

Ytbarek K, Abraham G, Girma T, Tilahun T, Woldie M (2020) The effect of bacillus Calmette-Guérin (BCG) vaccination in preventing severe infectious respiratory diseases other than TBC: implications for the COVID-19 pandemic. Vaccine 38(41):6374-6380

Wallin RPA, Lundqvist A, Moré SH, von Bonin A, Kiessling R, Ljunggren H-G (2002) Heat-shock proteins as activators of the innate immune system. Trends Immunol 23(3):130-135

Wang P, Sidney J, Dow C, Mothé B, Sette A, Peters B (2008) A systematic assessment of MHC class II peptide binding predictions and evaluation of a consensus approach. PLoS Comput Biol 4(4):e1000048.

Publisher's note Springer Nature remains neutral with regard to jurisdictional claims in published maps and institutional affiliations. 